\title{
Experimental Study of Discharge with Flexible Electrodes at Intermediate Pressure for Radar Cross Section Reduction
}

\author{
Received 23 August, 2021; revised 16 September, 2021; accepted 17 September, 2021
}

\author{
Jang Jae Lee ${ }^{a}$, Chul Hee Cho ${ }^{a}$, Min Su Choi ${ }^{a}$, Chang Seok Cho ${ }^{b}$, Tae Joo Oh ${ }^{b}$, Yong Shik Lee ${ }^{b}$, Jin Woo Yim ${ }^{c}$, \\ Heung Cheol Yoo ${ }^{c}$, Hee Soo Jung ${ }^{c}$, and Shin Jae You ${ }^{\mathrm{a}, \mathrm{d}, *}$ \\ a Applied Physics Laboratory for PLasma Engineering (APPLE), Department of Physics, Chungnam National Univer- \\ sity, Daejeon 34134, Republic of Korea \\ bDepartment of Electrical and Electronic Engineering, Yonsei University, Seoul 03722, Republic of Korea \\ ${ }^{c}$ Agency for Defense Development (ADD), Daejeon 34186, Republic of Korea \\ dInstitute of Quantum Systems (IQS), Chungnam National University, Daejeon 34134, Republic of Korea
}

*Corresponding author E-mail: sjyou@cnu.ac.kr

\begin{abstract}
In this experimental research, the characteristics of the discharge generated using a flexible electrode at intermediate pressure were investigated. We confirmed the discharge patterns according to the pulse width and frequency of a pulse generator with a fixed voltage under a pressure of $0.3 \mathrm{~atm}$, which is the pressure at a typical flight altitude. Uniform discharge was found to occur at a frequency of $10 \mathrm{kHz}$ with a pulse width of $4 \mu \mathrm{s}$ and at a frequency of $20 \mathrm{kHz}$ with a pulse width of $3 \mu \mathrm{s}$. To analyze the trend of discharge patterns, the voltage and current waveforms of the discharge in each condition were measured. In addition, the power dissipated by one cycle was calculated from the measured voltage and current waveforms. From the measurement and calculation results, it was confirmed that the dissipated power was large when the discharge occurred at a frequency of $20 \mathrm{kHz}$ with a pulse width of $3 \mu \mathrm{s}$, that is, the brighter and more uniform discharge pattern, which was found to be the optimal discharge condition for the flexible electrode at a pressure of $0.3 \mathrm{~atm}$.
\end{abstract}

Keywords: Flexible electrode, Intermediate pressure, Plasma, Radar cross section

\section{Introduction}

Plasma can be employed in stealth technology because of its wide absorption bandwidth and high absorption efficiency. The key principle is to surround the target with a plasma layer, which reflects or absorbs the incident radar signal, thereby reducing the radar cross section (RCS) as an indicator of the stealth capabilities of the target surrounded by the plasma layer. Based on this principle, many studies on RCS reduction using plasma have been conducted. Koretzky and Kuo demonstrated that plasma torches can effectively attenuate EM waves $[1,2]$. Wolf and Arjomandi measured the RCS by generating a dielectric barrier discharge at atmospheric pressure and calculated the attenuation effect of plasma using the Lorentz model to compare the measured RCS [3]. He et al. investigated electromagnetic attenuation numerically and experimentally using a semi-ellipsoidal shaped plasma layer [4]. Chayekhloo et al. observed significant electromagnetic wave dissipation in the frequency band using a plasma-arrayed coating structure [5]. Payne et al. developed an electrically tunable microwave absorber based on discrete plasma shells and analyzed its working principle using a transmission line model [6].

Although much research has been conducted to improve the process of RCS reduction by plasma, there are still problems that must be solved in order to apply plasma stealth technology to targets such as aircraft and aerospace vehicles. Most of the reported plasma sources for RCS reduction are rigid plates and cannot be used on curved surfaces. As a result, they generally cannot be applied to all surfaces on a target. Research on RCS reduction using plasma arrays applied to curved surfaces has been reported through simulation calculations [7], but experimental studies have not yet been reported. Therefore, it is necessary to use a component such as a flexible electrode as a source that can generate plasma even on a curved surface.

There have been many studies related to plasma using flexible electrodes. Cho and Kim developed a flexible sheet capable of generating plasma at atmospheric pressure and studied the discharge characteristics according to the applied voltage and dielectric thickness [8]. Kim et al. investigated the plasma characteristics of a floating electrodedielectric barrier discharge with flexible electrodes [9]. In addition, discharge using flexible electrodes has been used in various applications such as biotechnology [10,11], materials [12], and display devices [13]. However, there are no cases in which discharge using flexible electrodes has been used in the field for RCS reduction; in particular, flexible electrode discharge characteristics at medium pressures in which aircraft operate have not been reported.

In this study, the characteristics of the discharge generated using a flexible electrode at intermediate pressures were investigated. The discharge patterns according to the pulse width and frequency of a pulse generator with a fixed voltage under a pressure of $0.3 \mathrm{~atm}$, which is the pressure at a typical flight altitude of $30,000 \mathrm{ft}$ (approximately $9,000 \mathrm{~m}$ ) [14], were confirmed. In addition, the voltage and current waveforms of the discharge in each condition were measured, and the power dissipated by one cycle was calculated from the measured volt- 
(a)

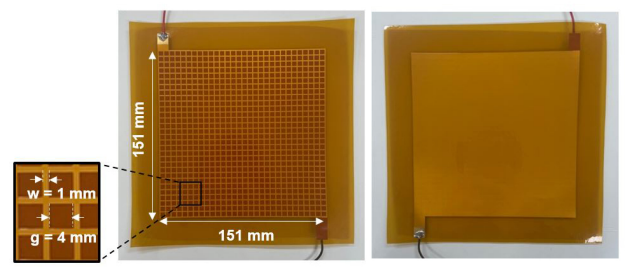

(b)

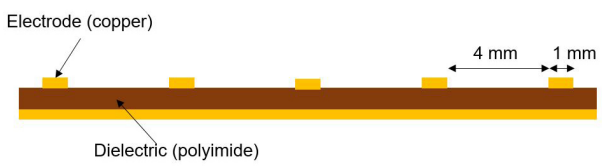

Figure 1. Flexible electrode (a) configuration and (b) cross section of the electrode structure.

age and current waveforms. From the measurement and calculation results, the effects of the pulse width and frequency on the discharge were analyzed.

\section{Experimental details}

Figure 1 shows the configuration of the flexible electrode used as the plasma source in our study. The flexible electrode consists of a 100 $\mu \mathrm{m}$ thick polyimide film with a meshed electrode printed on one side and a plane electrode on the other side. The electrodes are made of copper, and the mesh lines are $70 \mu \mathrm{m}$ thick and $1 \mathrm{~mm}$ wide, with 4 $\mathrm{mm}$ of spacing between adjacent lines.

A schematic of the experimental setup is shown in Fig. 2. To study the characteristics of discharge on the flexible electrode at intermediate pressure, the flexible electrode was placed inside a $20 \mathrm{~mm}$ thick acrylic cube with dimensions of $200 \mathrm{~mm} \times 200 \mathrm{~mm} \times 27 \mathrm{~mm}$. The pressure in the chamber was maintained at $0.3 \mathrm{~atm}$ using a rotary pump connected with an angle valve. A high-voltage pulse generator (IHP-1002, ITM) with an output voltage of $0-10 \mathrm{kV}$, a frequency of $0-40 \mathrm{kHz}$, and a pulse width of 2-5 $\mu$ s was used to generate the plasma on the flexible electrode. We used an oscilloscope (TDS3052A, Tektronix Inc.) connected to the experimental device with a voltage probe (TekP5100,

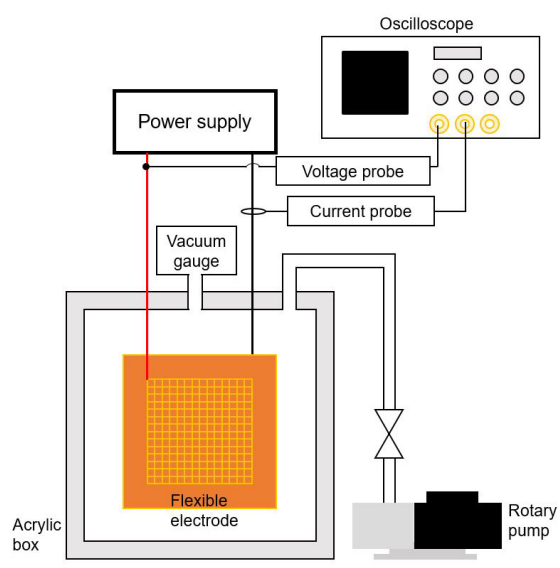

Figure 2. Schematic diagram of the experimental setup. (b)

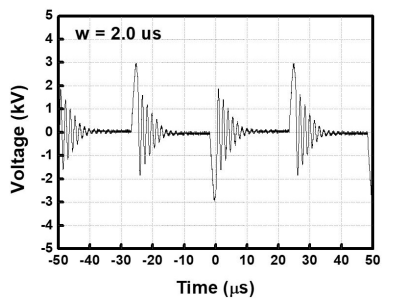

(c)

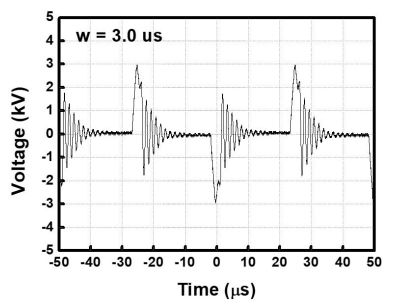

(d)
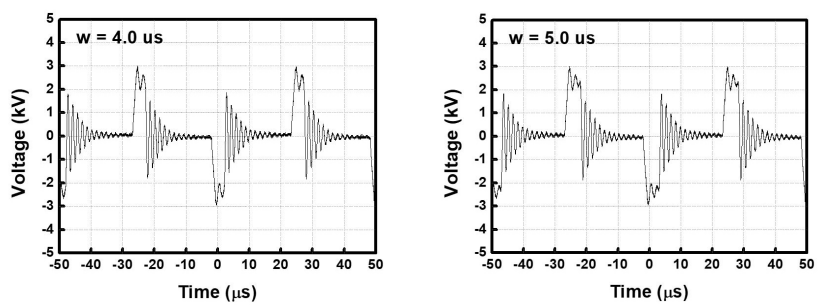

Figure 3. Voltage waveforms of the pulse generator according to the pulse width at an input voltage of $2.5 \mathrm{kV}$ with a frequency of $20 \mathrm{kHz}$ [Pulse width: (a) $2.0 \mu \mathrm{s}$, (b) $3.0 \mu \mathrm{s}$, (c) $4.0 \mu \mathrm{s}$, and (d) $5.0 \mu \mathrm{s}$.

Tektronix Inc.) and a current probe (TCP202, Tektronix Inc.) to measure the voltage and current waveforms during the experiments. Figure 3 shows the voltage waveform according to the pulse width of the generator used in this study at a fixed voltage of $2.5 \mathrm{kV}$ and a frequency of $20 \mathrm{kHz}$. Under this condition, the output voltage of the pulse generator was applied with a rise time of approximately $1.6 \mu$ s and a peak of $3.0 \mathrm{kV}$ during the set pulse width (on-time), and attenuated and oscillated with a decay time of approximately $4.0 \mu$ s during the off-time. It can be seen that some ringing occurred in the on-time range, and the ringing increased as the pulse width increased.

\section{Results and discussion}

Figure 4 shows the discharge patterns on the flexible electrode according to the pulse width $(w)$ and frequency $(f)$. The discharge patterns varied depending on the frequency and pulse width. At a short pulse width $(w=2 \mu \mathrm{s})$, no glow discharge occurred except at a frequency of $40 \mathrm{kHz}$. At $f=40 \mathrm{kHz}$, the discharge on the flexible electrode occurred even at a short pulse width $(w=2 \mu \mathrm{s})$, but a relatively localized discharge occurred compared to the long pulse width condition at the same frequency. At a frequency of $10 \mathrm{kHz}$, discharge occurred when the pulse width was $3 \mu$ s or more, and it can be seen that the larger the pulse width, the more uniform the discharge area. However, as mentioned above, the discharge pattern according to the pulse width changed with different frequencies. At $f=20 \mathrm{kHz}$, the discharge at the pulse width of $3 \mu \mathrm{s}$ was the brightest and most uniform, and as the pulse width increased, it became darker and the uniformity decreased. When the frequency was $30 \mathrm{kHz}$, there was almost no glow or uniformity in the discharge, and a wider discharge occurred at a pulse width of $3 \mu \mathrm{s}$ with the same frequency. At $40 \mathrm{kHz}$, as mentioned above, discharge occurred in all conditions, not just $w=2 \mu \mathrm{s}$, and discharge occurred in all areas of the flexible electrode, but showed a non-uniform discharge pattern.

To analyze the discharge pattern results, voltage and current waveforms under each condition were measured. Figure 5 shows the measured waveforms according to the pulse width at a frequency of 10 $\mathrm{kHz}$. The current waveform led the voltage waveform. When the voltage was applied ( $t=-7$ and $43 \mu \mathrm{s}$ ), the current rapidly increased and then gradually decreased. Subsequently, the current waveform rapidly decreased while oscillating during the off-time. The peak value of the voltage waveform during discharge was smaller than that of the input 
(a)

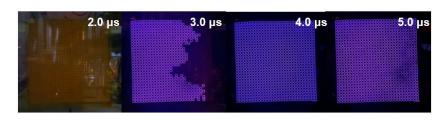

(c)
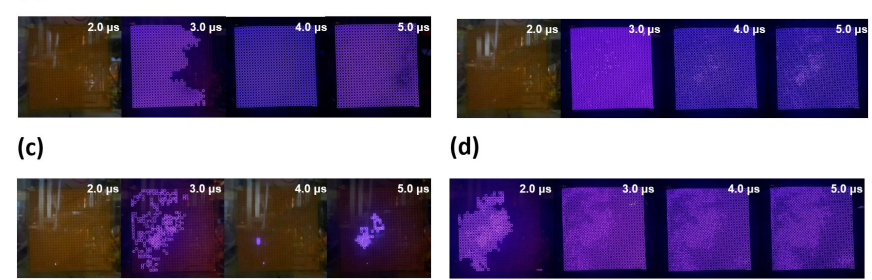

(d)

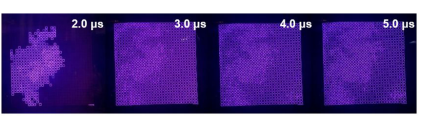

Figure 4. Discharge patterns in different pulse width and frequency with an input voltage of $3.0 \mathrm{kV}$ at a pressure of $0.3 \mathrm{~atm}$ [Frequency: (a) $10 \mathrm{kHz}$, (b) $20 \mathrm{kHz}$, (c) 30 $\mathrm{kHz}$, and $(\mathrm{d}) 40 \mathrm{kHz}]$

(a)

(b)

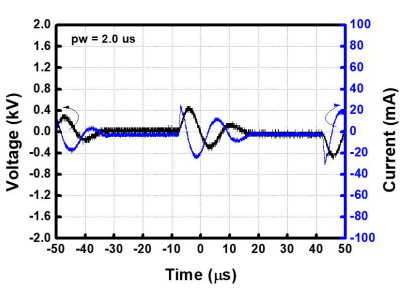

(c)

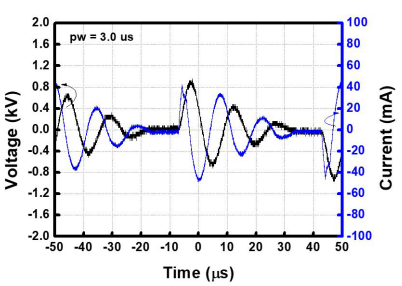

(d)
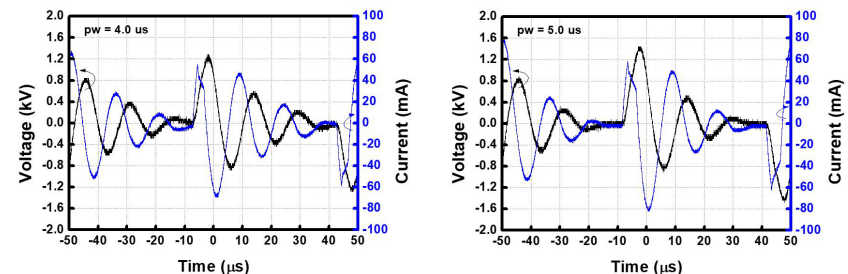

Figure 5. Measured voltage and current waveforms of discharge using the flexible electrode with an input voltage of $3.0 \mathrm{kV}$ and a frequency of $10 \mathrm{kHz}$ at a pressure of $0.3 \mathrm{~atm}$ [Pulse width: (a) $2.0 \mu \mathrm{s}$, (b) $3.0 \mu \mathrm{s}$, (c) $4.0 \mu \mathrm{s}$, and (d) $5.0 \mu \mathrm{s}$ ].

voltage waveform $(V=3.0 \mathrm{kV})$. This characteristic has been observed in previous studies $[15,16]$. As the rising time of the voltage increased owing to the discharge, the polarity of the voltage changed before the surface charges accumulated in the dielectric were sufficiently neutralized by controlling the driving frequency and pulse width of the pulse generator. Therefore, during discharge, the voltage waveform had a lower peak than the input voltage waveform. These waveforms explain the discharge phenomenon observed on the flexible electrode. When a voltage was applied to the flexible electrode, one side of the dielectric was charged and then discharged during a given pulse width section, after which the polarity was switched and charging and discharging occurred again. As this process was repeated, the magnitude of the current decreased, and the voltage measured at the flexible electrode, including discharge, also decreased along with the current. It can be seen that the amplitudes of voltage and current increased as the pulse width increased. This explains the tendency of the discharge pattern shown in Fig. 4(a). In addition, compared with the measured waveform in Fig. 5 and the discharge pattern in Fig. 4(a), it can be inferred that the breakdown voltage at which the glow discharge can occur in the flexible electrode at a pressure of 0.3 atm should be 0.8 $\mathrm{kV}$ or higher.

Figure 6 shows the measured voltage and current waveforms as a function of the pulse width at a frequency of $20 \mathrm{kHz}$. Compared with Fig. 4(b), as in the case of $f=10 \mathrm{kHz}$, a sudden increase in current occurred at the time of voltage application, after which it decreased through the discharge, and then damping oscillation occurred during the off-time. In addition, it can be seen that glow discharge occurred in the flexible electrode only when the voltage peak was $0.8 \mathrm{kV}$ or higher. (a)

(b)

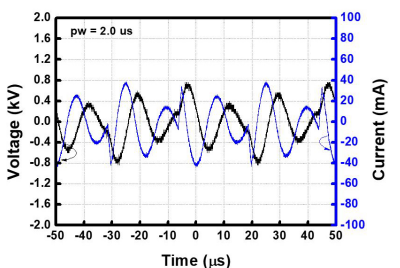

(c)

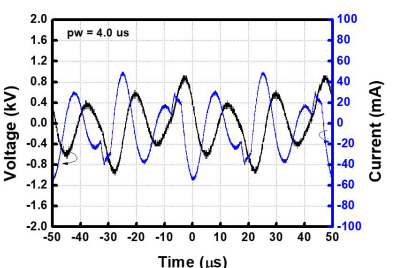

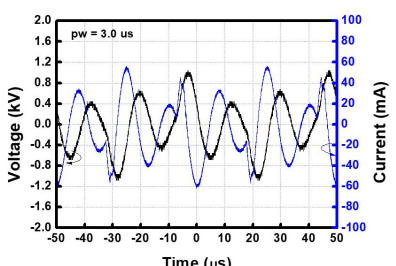

(d)

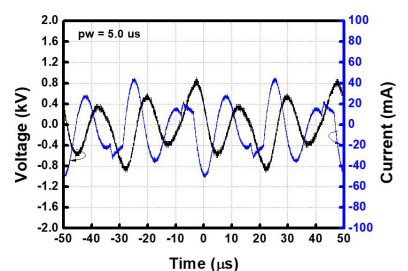

Figure 6. Measured voltage and current waveforms of discharge using the flexible electrode with an input voltage of $3.0 \mathrm{kV}$ and a frequency of $20 \mathrm{kHz}$ at a pressure of $0.3 \mathrm{~atm}$ [Pulse width: (a) $2.0 \mu \mathrm{s}$, (b) $3.0 \mu \mathrm{s}$, (c) $4.0 \mu \mathrm{s}$, and (d) $5.0 \mu \mathrm{s}$ ].

At $w=2 \mu \mathrm{s}$, the voltage peak was less than $0.8 \mathrm{kV}$ and no glow discharge occurred. However, it can be seen that the amplitude of the voltage and current waveforms was greatest at $w=3 \mu \mathrm{s}$, unlike when the frequency was $10 \mathrm{kHz}$, and the amplitude decreased as the pulse width increased. In addition, the current peak at the time at which the voltage was applied decreased with increasing pulse width. This behavior was consistent, which explains the tendency of the discharge pattern in Fig. 4(b).

When the frequency was $30 \mathrm{kHz}$, the measured voltage waveforms exhibited a complex waveform (Fig. 7). The voltage waveforms were similar in amplitude under all pulse width conditions and did not exceed $0.8 \mathrm{kV}$. Compared with Fig. 4(c), these results are interpreted to indicate that the glow discharge did not occur at $f=30 \mathrm{kHz}$ because the breakdown voltage for discharge on the flexible electrode at 0.3 atm was not exceeded. For the current waveforms, the magnitude of the current peaks decreased as the pulse width increased, such as at $f$ $=20 \mathrm{kHz}$.

(a)

(b)

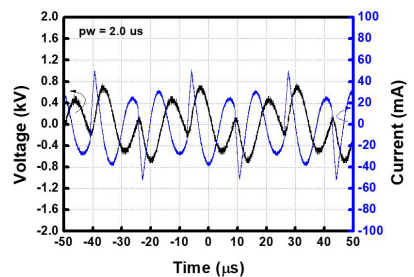

(c)

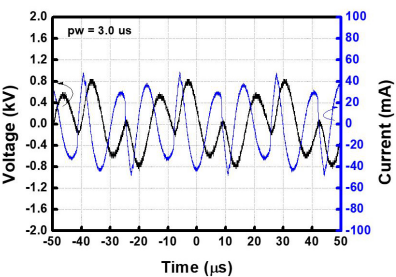

(d)
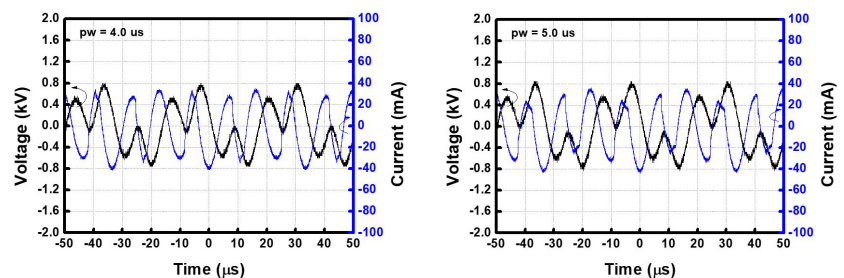

Figure 7. Measured voltage and current waveforms of discharge using the flexible electrode with an input voltage of $3.0 \mathrm{kV}$ and a frequency of $30 \mathrm{kHz}$ at a pressure of $0.3 \mathrm{~atm}$ [Pulse width: (a) $2.0 \mu \mathrm{s}$, (b) $3.0 \mu \mathrm{s}$, (c) $4.0 \mu \mathrm{s}$, and (d) $5.0 \mu \mathrm{s}$ ]. 
(b)

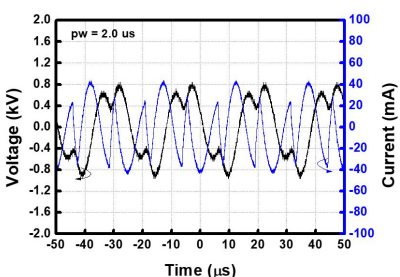

(c)

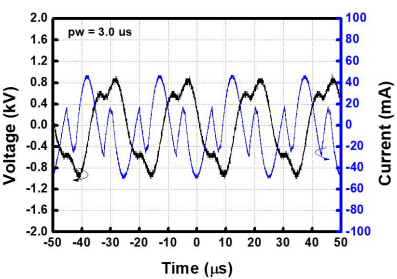

(d)

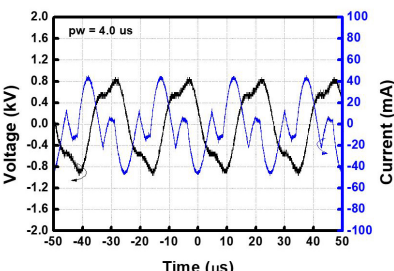

Figure 8. Measured voltage and current waveforms of discharge using the flexible electrode with an input voltage of $3.0 \mathrm{kV}$ and a frequency of $40 \mathrm{kHz}$ at a pressure of $0.3 \mathrm{~atm}$ [Pulse width: (a) $2.0 \mu \mathrm{s}$, (b) $3.0 \mu \mathrm{s}$, (c) $4.0 \mu \mathrm{s}$, and (d) $5.0 \mu \mathrm{s}$ ].

As shown in Fig. 8, the discharge voltage and current waveforms at $f=40 \mathrm{kHz}$ varied. At $w=2 \mu \mathrm{s}$, the voltage waveform exhibited a complex waveform similar to the voltage waveform at $f=30 \mathrm{kHz}$ However, it can be seen that as the pulse width increased, the slopes of the increasing voltage waveform and the damped oscillating waveform became increasingly similar at the time at which the voltage was applied (e.g. $t=-31,-6,19$, and $44 \mu \mathrm{s}$ ). In particular, at $w=5 \mu \mathrm{s}$, the voltage waveform was close to a square wave, and the current due to the discharge during the on-time period was almost constant with time. Therefore, it can be seen that brighter glow discharges were generated at $f=40 \mathrm{kHz}$ than at other frequency conditions [Fig. 4(d)].

Figure 9 shows the calculation of the power dissipated in the discharge according to the frequency with different pulse widths. The dissipated power was calculated as follows:

$$
P=\frac{1}{T} \int_{0}^{T} V(t) \cdot I(t) d t
$$

where $P$ is the calculated consumed power in watts $(\mathrm{W}), T$ is the period of the voltage in seconds, $V(t)$ is the voltage in volts, and $I(t)$ is the discharge current in amperes. As shown in Fig. 9, several $\mathrm{W}$ of power was dissipated in the plasma discharge, and a maximum of 12.7 $\mathrm{W}$ of power was consumed $(f=20 \mathrm{kHz}, w=3 \mu \mathrm{s})$. When the pulse width was short and the frequency was small, relatively little power was consumed, and the relationship between power and frequency varied according to the pulse width. At $w=2 \mu \mathrm{s}$, the dissipated power increased as the frequency increased, but this tendency did not occur when the pulse width increased. When the pulse width was $3 \mu \mathrm{s}$, the dissipated power had the largest value at $f=20 \mathrm{kHz}$, and there was little dependence on the frequency when the pulse width was 4 and $5 \mu \mathrm{s}$. Assuming that the dissipated power value calculated from the measured voltage and current waveforms contributes to the discharge, it can be seen that the condition of $f=20 \mathrm{kHz}$ and $w=3 \mu$ s is the optimal condition for generating a bright and uniform discharge on the flexible electrode at a pressure of $0.3 \mathrm{~atm}$.

\section{Conclusions}

The characteristics of discharge generated on a flexible electrode as a plasma source for RCS reduction were experimentally studied according to the frequency and pulse width of a pulse generator with a fixed voltage at a pressure of $0.3 \mathrm{~atm}$. When the pulse width was lower

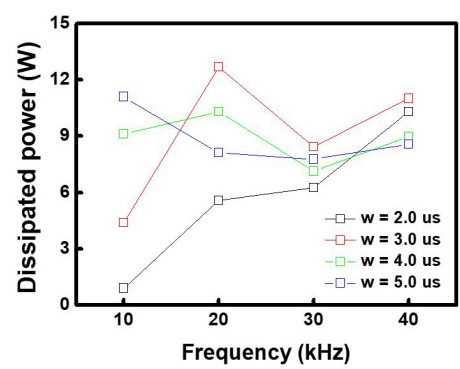

Figure 9. Calculated dissipated power with different frequencies and pulse widths in the discharge with an input voltage of $3.0 \mathrm{kV}$ at a pressure of $0.3 \mathrm{~atm}$.

( $w=2 \mu \mathrm{s}$ ) at a fixed frequency, glow discharge on the flexible electrode did not occur. However, discharge occurred locally at a higher frequency of the pulse generator $(f=40 \mathrm{kHz})$. When the pulse width was more than $3 \mu \mathrm{s}$, discharge occurred on the flexible electrode, but localized discharge occurred at a certain frequency of the pulse generator $(f=40 \mathrm{kHz})$. The discharge characteristics varied according to the frequency and pulse width. At a lower frequency $(f=10 \mathrm{kHz})$, the longer pulse widths resulted in a brighter and more uniform discharge. The amplitude of the voltage and current also increased with increasing pulse width. However, when the frequency was high, the dependency of the discharge on the pulse width disappeared. The brighter and more uniform discharge occurred at a frequency of $20 \mathrm{kHz}$ with a pulse width of $3 \mu \mathrm{s}$, which is the optimized condition for generating plasma on the flexible electrode used in this study. It was confirmed that the dissipated power calculated from the measured waveform also coincided with the tendency of the discharge pattern according to the frequency and pulse width. The results of this study are expected to be applied to achieve the RCS reduction effect using a flexible device in the future.

\section{Acknowledgements}

This research was supported by the Aerospace Low Observable Technology Laboratory Program of the Defense Acquisition Program Administration and the Agency for Defense Development of the Republic of Korea.

\section{Conflict of interest}

The authors declare no conflicts of interest.

\section{ORCID}

Jang Jae Lee

Chul Hee Cho

Min Su Choi

Chang Seok Cho

Tae Joo Oh

Yong Shik Lee

Jin Woo Yim

Heung Cheol Yoo

Hee Soo Jung

Shin Jae You https://orcid.org/0000-0002-0298-5638 https://orcid.org/0000-0002-6781-1057 https://orcid.org/0000-0001-7329-6250 https://orcid.org/0000-0002-9616-8072 https://orcid.org/0000-0003-3847-5557 https://orcid.org/0000-0003-2623-3569 https://orcid.org/0000-0002-9616-8072 https://orcid.org/0000-0003-3327-9944 https://orcid.org/0000-0002-8996-9007 https://orcid.org/0000-0002-8306-7643

\section{References}

[1] E. Koretzky and S. P. Kuo, Phys. Plasmas 5, 3774 (1998).

[2] S. P. Kuo, E. Koretzky, and L. Orlick, IEEE Trans. Plasma Sci. 27, 752 (1999).

[3] S. Wolf and M. Arjomandi, J. Phys. D: Appl. Phys. 44, 315202 (2011).

[4] X. He, J. Chen, Y. Zhang, Y. Chen, X. Zeng, and C. Tang, Plasma Sci. Technol. 17, 869 (2015). 
[5] A. Ghayekhloo, A. Abdolali, and S. H. M. Armaki, IEEE Trans. Antennas Propag. 65, 3058 (2017).

[6] K. Payne, K. Xu, J. H. Choi, and J. K. Lee, IEEE Trans. Antennas Propag. 67, 6523 (2019).

[7] S. H. Zainud-Deen, H. A. E-A. Malhat, and N. A. Shabayek, Plasmonics 15, 341 (2020).

[8] G. Cho and Y. Kim, Appl. Sci. Converg. Technol. 27, 23 (2018).

[9] J.-H. Kim, J.-S. Park, Y.-S. Shin, and C.-K. Kim, Korean J. Chem. Eng. 36, 1371 (2019).

[10] D. D. Jayasena, H. J. Kim, H. I. Yong, S. Park, K. Kim, W. Choe, and C. Jo, Food Microbiol. 46, 51 (2015).

[11] J. Kim, K.-H. Choi, Y. Kim, B. J. Park, and G. Cho, Appl. Sci. 7,
1308 (2017)

[12] Y. R. Lee, S. Lee, and D.-G. Kim, J. Korean Inst. Surf. Eng. 51, 133 (2018).

[13] R. S. Tarighat, A. Goodarzi, S. Mohajerzadeh, B. Arvan, M. R. Gaderi, and M. Fathipour, Proc. IEEE 93, 1374 (2005).

[14] S. Song et al., IEEE Trans. Plasma Sci. 49, 1548 (2021).

[15] J. J. Lee, S. J. Kim, Y. S. Lee, C. H. Cho, M. S. Choi, I. H. Seong, S. H. Lee, W. N. Jeong, and S. J. You, Appl. Sci. Converg. Technol. 29, 170 (2020).

[16] G. H. Kim and S. H. Song, J. Korean Phys. Soc. 49, 558 (2006). 